\title{
Semantic Representation of Patient Triage Data Collected in Emergency Departments
}

\author{
Evangelos LOGARAS ${ }^{\mathrm{a}}$, Antonis BILLIS ${ }^{\mathrm{a}, 1}$, Georgios PETRIDIS ${ }^{\mathrm{a}}$, Charalampos \\ BRATSAS $^{\mathrm{b}}$ and Panagiotis D. BAMIDIS ${ }^{\mathrm{a}}$ \\ ${ }^{a}$ Lab of Medical Physics, Medical School, Aristotle University of Thessaloniki, Greece \\ ${ }^{\mathrm{b}}$ School of Mathematics, Aristotle University of Thessaloniki, Greece
}

\begin{abstract}
Emergency Department (ED) overcrowding is a major issue for the efficient management of patients. To this end, triage algorithms have been developed to support the task of patient prioritization. In this paper an ontology was designed to represent the knowledge about patient triage procedure in EDs.
\end{abstract}

Keywords. Medical ontology, Emergency Department, Triage

\section{Introduction}

Triage is one of the fundamental processes in Emergency Departments (EDs) in order to prioritize individuals requiring urgent medical intervention. It is a complex decisionmaking process performed by the nursing staff based on patient's demographics, chief complaint, and vital signs. Several studies tried to tackle patient triaging in EDs using ontology-based systems, e.g. the OntolUrgences ontology [1]. This work focuses on the representation of the knowledge regarding patient triage procedure in the IntelTriage project [2]. To our knowledge, this is the first attempt to semantically model ED triage.

\section{Methodology and Results}

To design the ontology the collaborative approach was chosen [3]. The top-class Patient represents each patient incident. Information is divided into a set of five classes. Class Triage represents information related to the screening procedure. Class Medical represents patient's medical and social history. WaitingTime class represents the waiting time in different ED departments. Vitals class including subclasses HeartRate and OxygenSaturation represent heart rate (HR) and oxygen saturation (SpO2) measurements collected for each patient. Finally, patients' experienced symptoms are represented with the use of the Symptom class. The five classes, their subclasses and all the needed variables are presented in Fig1. In the context of semantic interoperability, an attempt was made to interconnect ontology's variables with common data models and other ontologies (SNOMED CT healthcare terminology [4], International Classification

\footnotetext{
${ }^{1}$ Corresponding Author, Antonis Billis, Lab of Medical Physics, Medical School, Aristotle University of Thessaloniki, Greece; E-mail: antonis.mpillis@gmail.com.
} 
of Primary Care (ICPC-2) [5]) that refer to the same field of interest. Following this process, the developed ontology is standardized, reusable and easy to interconnect.

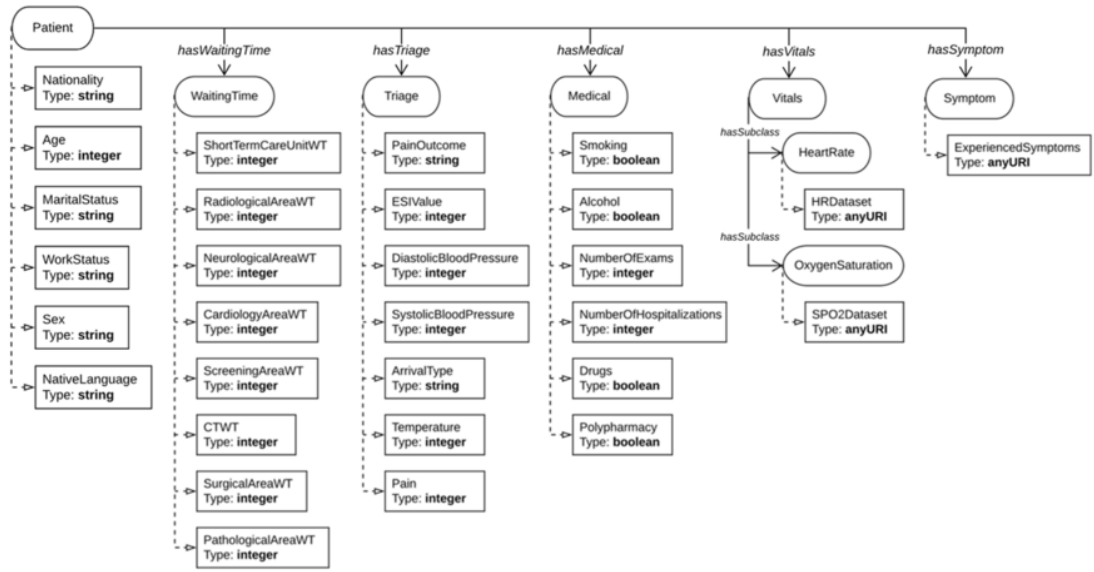

Figure 1. Ontology design.

\section{Conclusions}

The ontology presented in this paper is an effort to represent and share common understanding of the patient triage procedure in ED. Future steps include ontology validation and addition of real-life data to act as a data repository for ED incidents.

\section{Acknowledgments}

IntelTriage has been co-financed by the European Regional Development Fund of the EU and Greek national funds through the Operational Program Competitiveness, Entrepreneurship and Innovation, under the call Research-Create-Innovate (No. T1EDK02489).

\section{References}

[1] Charlet J, Declercka G, Dhombres F, Gayet P, Miroux P, Vandenbussche PY. Building a Medical Ontology to Support Information Retrieval: Terminological and Metamodelization Issues. Proceedings of the 10th International Conference on Terminology and Artificial Intelligence. Oct 28-30 2013;171-8.

[2] Billis A, Logaras E, Zouka M, Karanasiou N, Fourlis A, Nicopolitidis P, Lagakis P, Gialelis J, Kallergis D, Papadimitriou GI, Douligeris C, Papavramidis TS, Krizea M, Bamidis PD. Functional and nonfunctional requirements of a smart triage system for Emergency Departments: the case of IntelTriage project. 2019 4th South-East Europe Design Automation, Computer Engineering, Computer Networks and Social Media Conference (SEEDA-CECNSM); 2019; Piraeus, Greece. p. 1-4. doi: 10.1109/SEEDACECNSM.2019.8908320.

[3] Hristoskova A, Sakkalis V, Zacharioudakis G, Tsiknakis M, De Turck F. Ontology-driven monitoring of patient's vital signs enabling personalized medical detection and alert. Sensors (Basel). 2014 Jan 17;14(1):1598-628. doi: 10.3390/s140101598. PMID: 24445411; PMCID: PMC3926628.

[4] SNOMED Home. SNOMED International. (n.d.). Available at: https: //www.snomed.org/, Accessed March 8, 2021. 
[5] International Classification of Primary Care, 2nd edition (ICPC-2). (n.d.). Available at: https://www.who.int/standards/classifications/other-classifications/international-classification-ofprimary-care, Accessed March 8, 2021. 